Annali di Matematica pura ed applicata

(IV), Vol. CLXV (1993), pp. 261-279

\title{
A Universal Branching Set for 4-Dimensional Manifolds $\left({ }^{*}\right)\left({ }^{* *}\right)$.
}

\author{
MaRia Rita CASALI
}

Abstract. - In this work, a universal branching set $K$ for orientable 4-manifolds, such that

$$
\Pi_{1}\left(S^{4}-K\right)=\left\langle a, b, c / a c a^{-1} c^{-1}=1\right\rangle
$$

is proved to exist. This leads to the possibility of representing every closed connected orientable 4-manifold by a suitable transitive set $\{\sigma, \tau, \mu\}$ of permutations, in analogy with known results for dimension three (see [M] and [CV]).

\section{1. - Introduction.}

In [M], Montesinos shows the existence of a very simple universal branching set for 3-manifolds (the graph $G$ depicted in figure 3); since $\Pi_{1}\left(S^{3}-G\right)$ is a free group on two generators, this leads to the possibility of representing 3-manifolds by means of a transitive pair $\{\sigma, \tau\}$ of permutations in the symmetric group $S_{h}$ on $h$ elements, for some $h$. Later on, in [CV], this representation theory is related to the one making use of edge-coloured graphs; in particular, an algorithm is described to pass from a crystallization to a pair of permutations (one of which may be assumed to be of standard type) representing a chosen 3-manifold.

In this paper, we re-obtain the above results for dimension three by means of a standard method-described in [CG]—to yield edge-coloured graphs representing branched covering spaces, starting from the associated monodromy (section 3).

Further, in section 4, the same approch is used to face the analogous problems in dimension four: a universal branching set $K$ is obtained, such that $\Pi_{1}\left(S^{4}-K\right)$ admits a presentation $\left\langle a, b, c / a c a^{-1} c^{-1}=1\right\rangle$. Thus, every closed orientable 4-manifold $M^{4}$ is proved to be representable by a suitable transitive set $\{\sigma, \tau, \mu\}$ of permutations, such

(*) Entrata in Redazione il 26 Marzo 1991.

Indirizzo dell'A.: Dipartimento di Matematica Pura ed Applicata «G. Vitali», Via Campi 213 B, Università di Modena, I-41100 Modena (Italy).

(**) Work performed under the auspicies of the G.N.S.A.G.A. of the C.N.R. (National Research Council of Italy) and financially supported by MURST (project «Geometria Reale $\mathrm{e}$ Complessa»). 
that $\sigma \mu \sigma^{-1} \mu^{-1}=1$ (Corollary 10); moreover, the permutations $\sigma$ and $\tau$ may always be assumed to be of standard type, so that the only permutation $\mu$ is sufficient to reconstruct the 4-manifold (Proposition 11).

A glance towards higher dimensions concludes the paper: every closed orientable $n$-manifold $M^{n}(n \geqslant 5)$ is shown to be representable by a suitable transitive set $\left\{\sigma_{1}, \sigma_{2}, \ldots, \sigma_{2^{n-3}}, \sigma_{2^{n-3}+1}\right\}$ of permutations (among which only one is essential), constituting the monodromy with respect to a universal branching set (section 5).

\section{2. - Preliminaries.}

Throughout this paper, all spaces and maps are piecewise-linear (PL), in the sense of [Gl] or [RS]. Manifolds are always assumed to be connected and orientable. We will often identify a ball complex with its asociated polyhedron and every homeomorphic space. For basic graph theory we refer to [W].

An edge-coloration on a multigraph $\Gamma=\left(V(\Gamma), E(\Gamma)\right.$ is a map $\gamma: E(\Gamma) \rightarrow \Delta_{n}=$ $=\{0,1, \ldots, n\}$ such that $\gamma(e) \neq \gamma(f)$, for each pair $e, f$ of adjacent edges. The pair $(I, \gamma)$, $\Gamma$ being a regular multigraph of degree $n+1$ and $\gamma: E(\Gamma) \rightarrow \Delta_{n}$ being an edge-coloration, is said to be an $(n+1)$-coloured graph. For each $B \subseteq \Delta_{n}$, we set $I_{B}\left(V(I), \gamma^{-1}(B)\right)$; each connected component of $\Gamma_{B}$ is often called a $B$-residue. An $m$ residue is a $B$-residue such that the cardinality of $B$ is $m$. The symbol $g_{B}$ denotes the number of $B$-residues. For each colour $c \in \Delta_{n}$, we set $\hat{c}=\Delta_{n}-\{c\}$.

An $n$-dimensional labelled pseudocomplex $K(\Gamma)$ (see [HW] and [BM]) can be associated with any $(n+1)$-coloured graph $(I, \gamma)$, via the following construction:

i) for each $v \in V(\Gamma)$, take an $n$-simplex $\sigma(v)$, with its vertices labelled by $0,1, \ldots, n$;

ii) for each $e \in E(\Gamma)$ with end-points $v, w \in V(\Gamma)$ and colour $\gamma(e)=c$, identify the $(n+1)$-faces of $\sigma(v)$ and $\sigma(w)$ opposite to the vertex labelled by $c$, so that equally labelled vertices coincide. dron.

We say that $(\Gamma, \gamma)$ represent $K(\Gamma),|K(T)|$ and every homeomorphic polyhe-

Note that every $\left\{c_{1}, \ldots, c_{m}\right\}$-residue $\Xi$ of $T$ uniquely corresponds to a $(n-m)$ simplex $\sigma^{n-m}$ of $K(\Gamma)$, whose vertices are labelled by $\Delta_{n}-\left\{c_{1}, \ldots, c_{m}\right\}$.

Moreover, it is known (see [FGG]) that:

A) $K(\Gamma)$ is orientable iff $\Gamma$ is a bipartite graph;

$B) K(\Gamma)$ is a (closed) $n$-manifold iff, for every $n$-residue $\Xi$ of $\Gamma, K(E)$ is homeomorphic to the $(n-1)$-sphere $S^{n-1}$.

A crystallization of a closed $n$-manifold $M^{n}$ is an $(n+1)$-coloured graph $(\Gamma, \gamma)$ representing $M^{n}$ such that $\Gamma_{\tilde{c}}$ is connected for each $c \in \Delta_{n}$. A theorem of [P] ensures the existence, for every closed $n$-manifold $M^{n}$, of crystallizations of $M^{n}$ (and hence of 
$(n+1)$-coloured graphs representing $\left.M^{n}\right)$; see also [FGG] and its bibliography for successive extensions of this result.

If $(\Gamma, \gamma)$ is an $(n+1)$-coloured graph representing a closed $n$-manifold $M^{n}$, and $\Gamma_{\hat{c}}$ is connected, for some $c \in \Delta_{n}$, it is known [Gr] that a presentation $\langle X / R\rangle$ of the fundamental group $\Pi_{1}\left(M^{n}\right)$, called c-edge presentation, can be obtained in the following way:

*) the generators of $X$ are the c-coloured edges, arbitrarily oriented;

**) the relators of $R$ are obtained by walking along the (arbitrarily oriented) 2residues of $\Gamma_{\{c, d\}}$, for each $d \in \Delta_{n}-\{c\}$, giving the exponent +1 or -1 to each generator whether the orientation of the 2-residue is coherent or not with the orientation of the generator.

Note that, if $\Gamma_{\hat{c}}$ is not connected, the $c$-edge presentation of $\Pi_{1}(M)$ can be obtained in a similar way: it is sufficient to complete the relators of $R$ with a minimal set of generators such that the corresponding $c$-coloured edges connect $\Gamma_{\bar{c}}$.

Let $(I, \gamma),\left(\Gamma^{\prime}, \gamma^{\prime}\right)$ be $(n+1)$-coloured graphs. For a positive integer $m(1 \leqslant m \leqslant$ $\leqslant n$ ), a map $f: V\left(\Gamma^{\prime}\right) \rightarrow V(T)$ is said to be an $m$-covering [V] if $f$ preserves $c$-adjacency for all $c \in \Delta_{n}$ and is bijective when restricted to $m$-residues. The branching $(m+1)$ residues are the $(m+1)$-residues of $(T, \gamma)$ covered by at least one $(m+1)$-residue of $\left(\Gamma^{\prime}, \gamma^{\prime}\right)$ on which $f$ is not injective.

The covering $f$ naturally induces a topological map $|f|: K\left(\Gamma^{\prime}\right) \rightarrow K(\Gamma)$. An $n$-covering induces a topological covering between the underlying topological space, while a 1-covering induces a topological covering branched over the $(n-2)$-subcomplex of $K(\Gamma)$ whose $(n-2)$-simplexes are represented by the branching 2-residues of $(\Gamma, \gamma)$.

Let $(\Gamma, \gamma)$ be an $(n+1)$-coloured graph representing a closed (orientable) $n$-manifold $K\left(T^{\prime}\right)=M^{n}$. Suppose $\Gamma_{\bar{c}}$ connected and let $L$ be the $(n-2)$-subcomplex of $K(T)$ represented by a given set $\left\{C_{1}, C_{2}, \ldots, C_{p}\right\}$ of 2-residues containing the colour $c$. In this hypothesis we say that $(K(\Gamma), L)$ is a branched $(n+1)$-coloured pair (with respect to the colour $c)$.

If $P=\left\langle X=\left\{x_{1}, \ldots, x_{s}\right\} / R=\left\{r_{1}, \ldots, r_{t}\right\}\right\rangle$ is a $c$-edge presentation of the fundamental group $\Pi_{1}\left(M^{n}\right)$ and if $r_{t-p+1}, \ldots r_{t}$ are the relators obtained by reading the 2residues $C_{1}, \ldots C_{p}$, then it is easy to check that $P^{\prime}=\left\langle X / R^{\prime}=\left\{r_{1}, \ldots, r_{t-p}\right\}\right\rangle$ is a presentation (called again c-edge presentation) of $I_{1}\left(M^{n}-L\right)$.

The existence of a one-to-one correspondence $\Phi$ between transitive representations $\omega$ of $\Pi_{1}\left(M^{n}-L\right)$ in $S_{d}$ (the symmetric group on the set $N_{d}=\{1,2, \ldots, d\}$ ) and $d$-fold covering space of $M^{n}$ branched over $L$, is well-known (see [F]). In [CG], the following method is desribed for constructing an $(n+1)$-coloured graph $(\tilde{\Gamma}, \tilde{\gamma})$ sich that $K(\tilde{\Gamma})=\Phi(\omega)$ :

$$
- \text { set } V(\widetilde{\Gamma})=V(\Gamma) \times N_{d} ;
$$

- for each $k \in \Delta_{n}-\{c\}$ and $i \in N_{d}$, join $(v, i)$ with $(w, i)$ by a $k$-coloured edge if $v, w$ are $k$-adjacent in $(T, \gamma)$; 
- join $(v, i)$ with $(w, j)$ by a $c$-coloured edge if in $(\Gamma, \gamma)$ there is an oriented $c$ coloured edge $x_{l}$ from $v$ to $w$ and $w\left(x_{l}\right)(i)=j$.

Roughly speaking, $(\tilde{\Gamma}, \tilde{\gamma})$ is obtained by taking $d$ copies of the (connected) $n$ coloured graph $\Gamma_{\hat{c}}$ and by drawing the $c$-coloured edges in the way induced by the permutations of $\omega(X)$.

\section{3. - Re-obtaining known results in dimension three.}

Let $(\Lambda, \lambda)$ be a crystallization of a closed connected orintable 3-manifold $M^{3}$, and let $V^{\prime}(\Lambda), V^{\prime \prime}(\Lambda)$ be the two bipartition classes of its vertex set. Further, let $v=$ $=\left(\cup_{0}, v_{1}, v_{2}, v_{3}\right)$ be an arbitrarily fixed permutation of the colour set; in the following, for sake of notational simplicity, we shall always write $i$ instead of $v_{i}$, for every $i \in \Delta_{3}$.

Let $(\tilde{\Lambda}, \tilde{\lambda})$ be the 4-coloured graph representing $M^{3}$ such that the pseudocomplex $K(\bar{\Lambda})$ is obtained from $K(\Lambda)$ by baricentrically subdividing the 2-simplex opposite to the (unique) 3-labelled vertex $v_{3}$, and then by starring from $v_{3}$ itself. It is easy to check that $(\tilde{\Lambda}, \tilde{\lambda})$ can directly be obtained from $(\Lambda, \lambda)$ by means of the following rules:

- for every $v \in V(\Lambda)$, construct a copy of the 2-coloured cycle $(H, h)$ of figure 1 , and label its vertices by the elements of the set $N_{6} \times\{v\}$, so that the vertex $(i, v)$ is the copy of the vertex $i$ of $(H, h)$;

- for every $i \in\{1,2\}$ (resp. $i \in\{3,4\}$ ) (resp. $i \in\{5,6\}$ ), connect $(i, v)$ with $(i, w)$ by a 2 -coloured edge iff $v$ and $w$ are connected in $(\Lambda, \lambda)$ by a 0 -coloured (resp. 1-coloured) (resp. 2-coloured) edge;

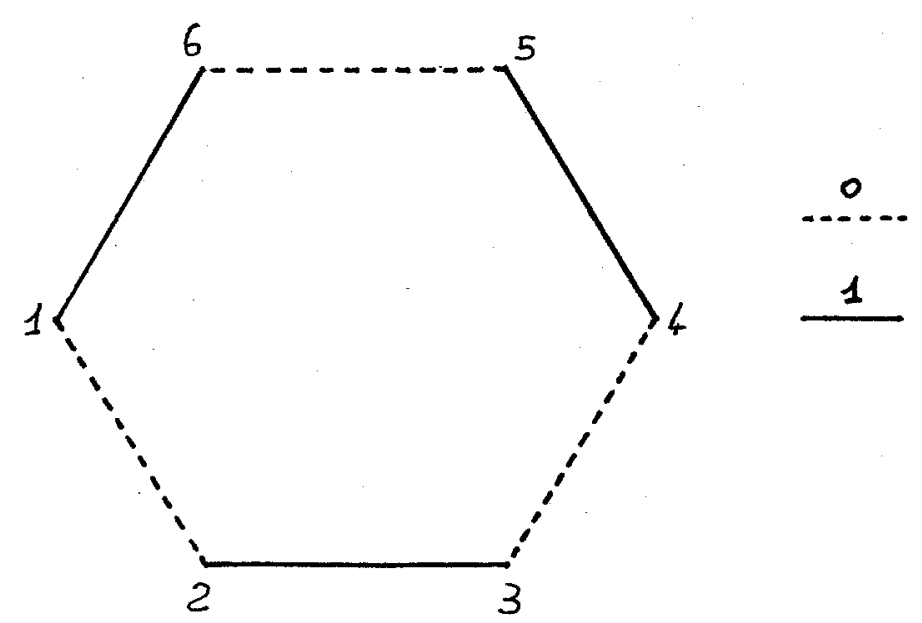

Fig. 1. 

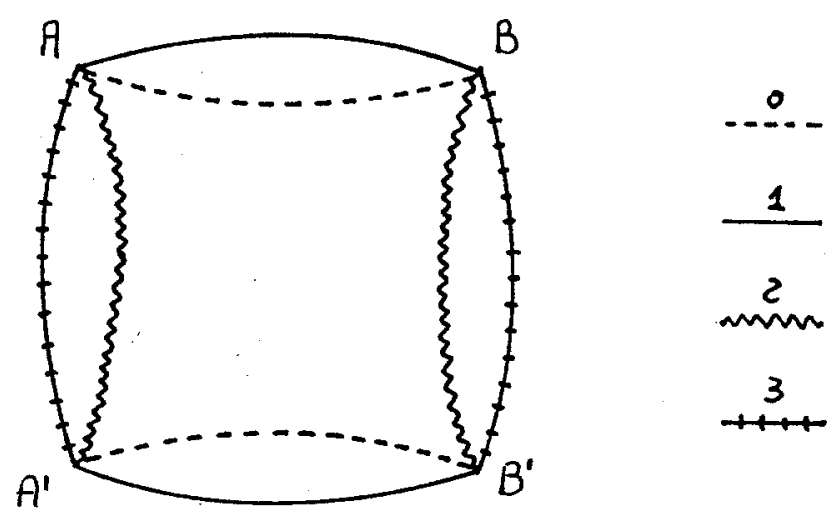

Fig. 2.

- for every $i \in N_{6}$, connect $(i, v)$ with $(i, w)$ by a 3-coloured edge iff $v$ and $w$ are connected in $(\Lambda, \lambda)$ by a 3 -coloured edge.

Let now $(\bar{\Lambda}, \bar{\lambda})$ denote the standard 4-coloured graph of figure 2, which evidently represents $S^{3}$. An easy direct computation proves the following result.

Proposition 1. - Let $g:(\tilde{\Lambda}, \tilde{\lambda}) \rightarrow(\bar{\Lambda}, \bar{\lambda})$ be the map defined by

$$
g(i, v)= \begin{cases}A & \text { if } i \text { even and } v \in V^{\prime}(\Lambda), \\ B & \text { if } i \text { odd and } v \in V^{\prime}(\Lambda), \\ A^{\prime} & \text { if } i \text { even and } v \in V^{\prime \prime}(\Lambda), \\ B^{\prime} & \text { if } i \text { odd and } v \in V^{\prime \prime}(\Lambda)\end{cases}
$$

Then, $g$ is a 1-covering, branched over the 2-residues of colours $\{0,1\},\{1,2\}$, $\{2,3\}$.

The 1-covering $g$ naturally induces a topological covering $|g|: M^{3} \rightarrow S^{3}$, branched over the subcomplex $G$ of $K(\bar{\Lambda})$ represented by the branching 2 -residues of $(\bar{\Lambda}, \bar{\lambda})$. It is now easy to check, by analizing the pseudosimplicial structure of $K(\bar{\Lambda})$, that the above defined subcomplex $G$ is exactly the graph depicted in figure 3 (already proved to be a universal branching set for 3 -manifolds in $[\mathrm{M}]$ ).

COROLlaRY 2. - Every closed connected orientable 3-manifold $M^{3}$ is a covering of $S^{3}$ branched over the universal set $G$.

Since branched covering spaces are uniquely determined by the monodromy of the covering map $[\mathrm{F}]$, we are now going to determine the group of $G$, i.e. $\Pi_{1}\left(S^{3}-G\right)$.

For, we consider the (unique) bisection $\bar{\Lambda}^{\prime}$ of type $(0,2)$ of $\bar{\Lambda}$ (see [G]); $\bar{\Lambda}^{\prime}$ (which is drawn in figure 4) represents a pseudocomplex $K\left(\bar{\Lambda}^{\prime}\right)$ triangulating $S^{3}$, such that 


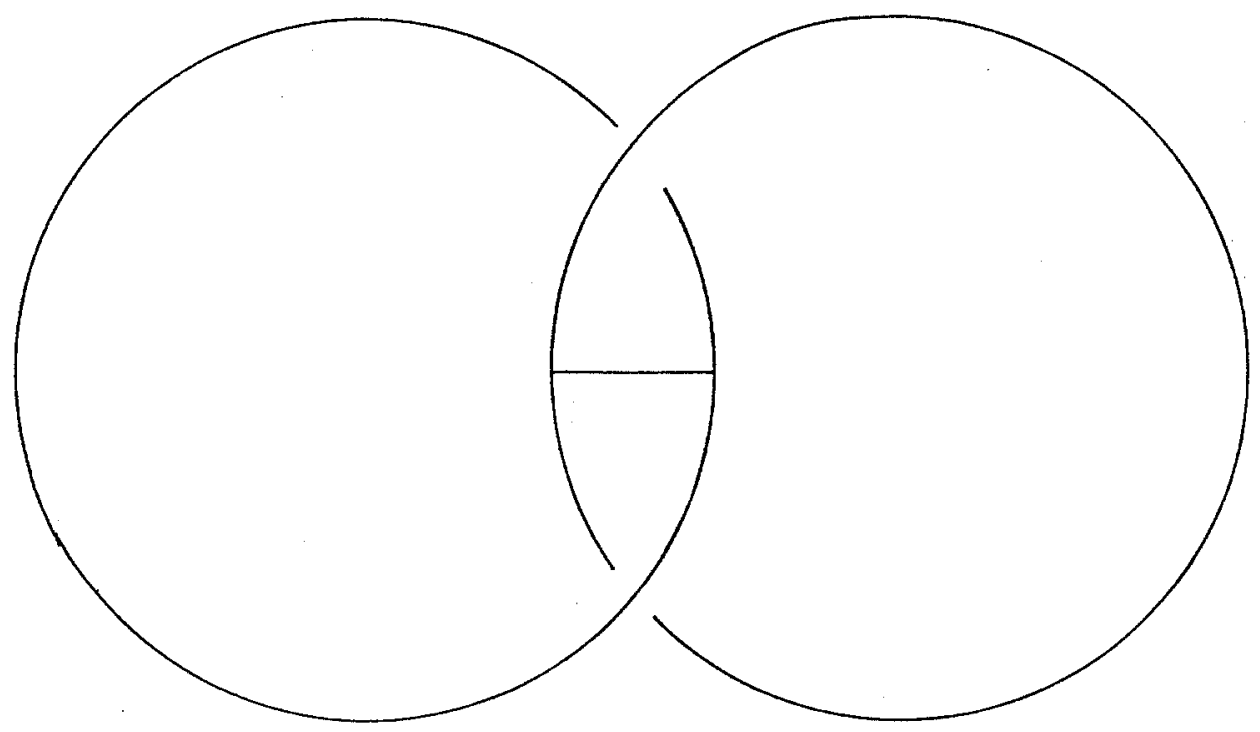

Fig. 3.

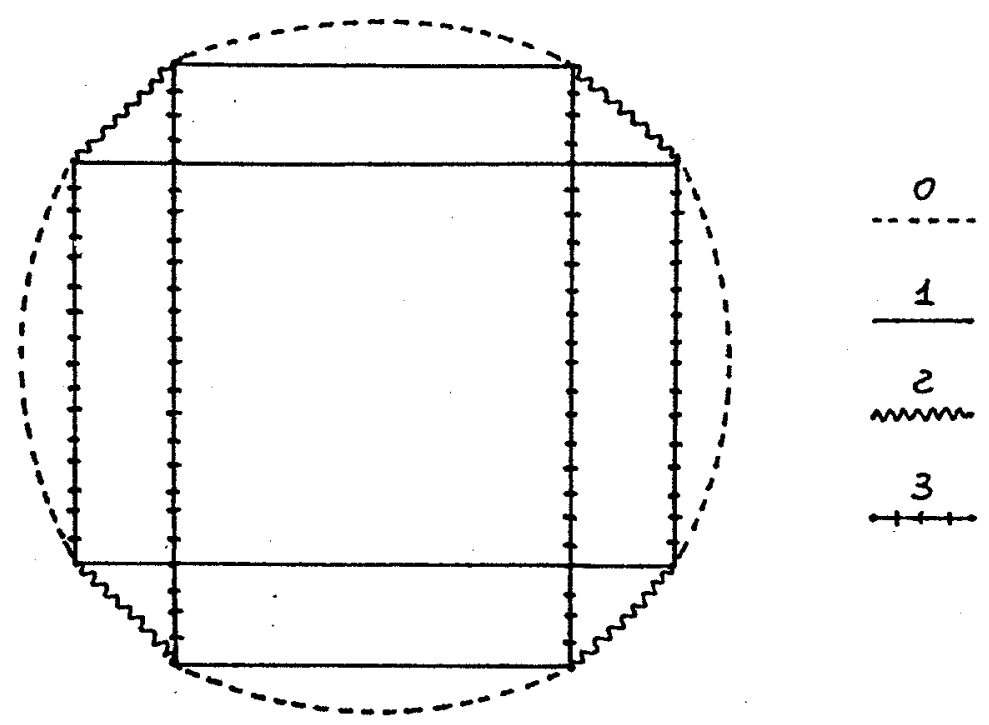

Fig. 4.

$\left(K\left(\bar{\Lambda}^{\prime}\right), G\right)$ is a branched 4-coloured pair (with respect to the colour 0 ). Thus, it is possible to obtain $\Pi_{1}\left(S^{3}-G\right)$ from the 0 -edge presentation of $\Pi_{1}\left(S^{3}\right)$ associated to $\bar{\Lambda}^{\prime}$.

Proposition 3. $-I_{1}\left(S^{3}-G\right)=\langle a, b /\rangle$.

Proof. - As pointed out in section $2, \Pi_{1}\left(S^{3}-G\right)$ is obtained from the 0-edge presentation of $\Pi_{1}\left(S^{3}\right)$ associated to $\bar{A}^{\prime}$ by deleting all the relators corresponding to 
branching 2-residues (i.e. to 1-simplices of $K\left(\bar{\Lambda}^{\prime}\right)$ belonging to the subcomplex $G$ ). Hence,

$$
\begin{aligned}
& \Pi_{1}\left(S^{3}-G\right)=\left\langle x_{1}, x_{2}, x_{3}, x_{4} / x_{1} x_{2} x_{3} x_{4}=1, x_{1} x_{3}=1\right\rangle= \\
& \quad=\left\langle x_{1}, x_{2}, x_{4} / x_{1} x_{2} x_{1}^{-1} x_{4}=1\right\rangle=\left\langle x_{1}, x_{2} / x_{1} x_{2} x_{1}^{-1} x_{1} x_{2}^{-1} x_{1}^{-1}=1\right\rangle=\left\langle x_{1}, x_{2} /\right\rangle .
\end{aligned}
$$

COROLLARY 4. - Every closed connected orientable 3-manifold $M^{3}$ is represented by a suitable transitive set $\{\sigma, \tau\}$ of permutations.

Proof. - If $\omega: I_{1}\left(S^{3}-G\right) \rightarrow S_{d}$ is the monodromy associated to the covering map from $M^{3}$ to $S^{3}$ branched over $G$, then it is sufficient to set $\sigma=\omega(a), \tau=\omega(b), a, b$ being the generators of the presentation of $\Pi_{1}\left(S^{3}-G\right)$ obtained in Prop. 3.

We want now to analize the algebraic features of the set $\{\sigma, \tau\}$ of permutations associated to a given 3-manifold $M^{3}$, by computing the monodromy of the topological covering $|q|$ induced by the 1-covering $g$ of Prop. 1. For, we consider the (global) bisection $\bar{\Lambda}^{\prime}$ of type $(0,2)$ of $\bar{\Lambda}$, so that the 1-covering $g$ between $\bar{A}$ and $\bar{\Lambda}$ naturally induces a 1-covering $g^{\prime}$ between the graph $\widetilde{\Lambda}^{\prime}$ (representing $M^{3}$ ) and the graph $\bar{\Lambda}^{\prime}$ (representing $S^{3}$ ). It is now easy to check that the 1-covering $g^{\prime}: \bar{\Lambda}^{\prime} \rightarrow \bar{\Lambda}^{\prime}$ can be obtained as an application of a standard method-due to [CG] and briefly described in section 2-to yield coloured graphs representing branched coverings of the sphere, starting from the associated monodromy. Since $\widetilde{\Lambda}^{\prime}$ contains three copies of $\bar{\Lambda}_{2}^{\prime}$ for every pair $\{v, w\}$ of 3 -adjacent vertices of $(\Lambda, \lambda), g^{\prime}$ induces a topological $(3 \cdot q / 2)$-fold covering $(q=\# V(\Lambda))$, whose monodromy is described by the 0 -coloured edges connecting the various sheets.

Thus, $\sigma, \tau$ may be thought of as permutations on the set $T=N_{3} \times N_{q / 2}$, by denoting $V^{\prime}(\Lambda)=\{1,2, \ldots, q / 2\}$ and $V^{\prime \prime}(\Lambda)=\left\{1^{\prime}, 2^{\prime}, \ldots, q^{\prime} / 2\right\}$, with $i$ 3-adjacent to $i^{\prime}$ for every $i \in N_{q / 2}$; moreover, the combinatorial properties of the graph $\tilde{\Lambda}^{\prime}$ ensure that $\sigma$ may always be assumed to be of standard type (only depending on $q=\# V(\Lambda)$ ), while $\tau$ directly depends on the combinatorial properties of the starting crystallization $(\Lambda, \lambda)$ of $M^{3}$ :

$$
\sigma=\prod_{i=1}^{q / 2}((1, i)(2, i)(3, i))
$$

$(*) \quad \tau((x, i))=\left(x, \tau_{j(x)}(i)\right), \quad$ where $j(x)= \begin{cases}0 & x \in\{1,2\}, \\ 1 & x \in\{3,4\}, \\ 2 & x \in\{5,6\},\end{cases}$

and $\tau_{j}(i)(j \in\{0,1,2\})$, for every vertex $i \in V^{\prime}(\Lambda)$, is the vertex $r \in V^{\prime}(\Lambda)$ which is 3 -adjacent to the vertex $r^{\prime} \in V^{\prime \prime}(\Lambda) j$-adjacent to $i$.

Note that $\{\sigma, \tau\}$ is obviously a transitive set, since $\widetilde{A}^{\prime}$ is a connected graph.

Further, we point out that the only permutation $\tau$ on $T$ is sufficient to reconstruct 
$(\Lambda, \lambda)$. In fact, $(\Lambda, \lambda)$ is isomorphic to the 4-coloured graph $\Lambda(\tau)$ obtained by the following rules:

- the vertex set of $\Lambda(\tau)$ is $\{1,2, \ldots q / 2\} \cup\left\{1^{\prime}, 2^{\prime}, \ldots q^{\prime} / 2\right\}$;

- every vertex $i \in\{1,2, \ldots q / 2\}$ is connected with the homonymous vertex $i^{\prime} \in\left\{1^{\prime}, 2^{\prime}, \ldots q^{\prime} / 2\right\}$ by a 3-coloured edge;

- every vertex $i \in\{1,2, \ldots q / 2\}$ is connected with the vertex $\left(\tau_{j}(i)\right)^{\prime} \in$ $\in\left\{1^{\prime}, 2^{\prime}, \ldots q^{\prime} / 2\right\}$ by a $j$-coloured edge, for every $j \in\{0,1,2\}$.

Thus, we have described an algorithm for obtaining, from any 3-manifold $M^{3}$-given by a crystallization $(\Lambda, \lambda)$-, a permutation $\tau$ which completely represents it; though essentially different, this algorithm gives the same result as the one described in [CV].

Proposition 5. - Every closed connected orientable 3-manifold $M^{3}$ is represented by a suitable permutation $\tau$ on $T=N_{3} \times N_{q / 2}$ such that

$$
\pi_{1} \circ \tau=1 \quad \text { and } \quad\left(\pi_{2} \circ \tau\right)((x, i))= \begin{cases}\tau_{0}(i) & x \in\{1,2\}, \\ \tau_{1}(i) & x \in\{3,4\}, \\ \tau_{2}(i) & x \in\{5,6\},\end{cases}
$$

(or, equivalently, by a suitable set $\left\{\tau_{0}, \tau_{1}, \tau_{2}\right\}$ of permutations on $N_{q / 2}$ ).

REMARK. - The three permutations $\tau_{0}, \tau_{1}, \tau_{2}$ on $N_{q / 2}$ correspond to the rows of the «code» for 3-manifolds, defined in [LD]. Thus, the conditions obtained in [CV] for deciding whether a given set $\left\{\tau_{0}, \tau_{1}, \tau_{2}\right\}$ of permutations represents a 3 -manifold $M^{3}$ or not, may be used to solve the analogous problem for «codes».

If $\mathscr{T}=\left\{\tau_{0}, \tau_{1}, \tau_{2}\right\}$ is a set of permutations representing a closed 3-manifold $M^{3}$, then the crystallization $\Lambda(\tau)$ of $M^{3}$, $\tau$ being obtained from $\left\{\tau_{0}, \tau_{1}, \tau_{2}\right\}$ by formula $(*)$, is said to be canonically associated to $\mathscr{T}$.

The following result shows how easily $\mathscr{T}=\left\{\tau_{0}, \tau_{1}, \tau_{2}\right\}$ may produce the fundamental group of the represented 3-manifold, via the 3-edge presentation of the canonically associated crystallization.

Proposition 6. - Let $\mathscr{T}=\left\{\tau_{0}, \tau_{1}, \tau_{2}\right\}$ be a set of permutations on $N_{q / 2}(q \in N)$, representing a closed 3-manifold $M^{3}$. Then, a (positive) presentation of $\Pi_{1}\left(M^{3}\right)$ is

$$
\left\langle X / R_{0} \cup R_{1} \cup R_{2}\right\rangle,
$$

where the set $X=\left\{x_{1}, x_{2}, \ldots, x_{q / 2}\right\}$ of generators is in bijection with $N_{q / 2}$, and the set $R_{i}=\left\{r_{i}^{1}, r_{i}^{2}, \ldots r_{i}^{\sigma_{i}}\right\}$ of relators $\left(i \in \Delta_{2}\right)$ is in bijection with the set $\left\{c_{i}^{1}, c_{i}^{2}, \ldots c_{i}^{\sigma_{i}}\right\}$ of disjoint cycles of the permutation $\tau_{i}, r_{i}^{s}\left(s=1,2, \ldots, \sigma_{i}\right)$ being obtained by juxtapposing the generators corresponding to the ordered elements of $c_{i}^{\varepsilon}$. 
Proof. - Let $\Lambda=\Lambda(\tau)$ be the crystallization of $M^{3}$ canonically associated to the set $\mathcal{T}$ of permutations. By construction, the set of 3-coloured edges of $A$ is $\left\{x_{1}, x_{2}, \ldots, x_{q / 2}\right\}, i \in\{1,2, \ldots, q / 2\}$ and $i^{\prime} \in\left\{1^{\prime}, 2^{\prime}, \ldots, q / 2\right\}$ being the end-points of $x_{i}$, for every $i \in\{1,2, \ldots, q / 2\}$. If every edge $x_{i}(i \in\{1,2, \ldots, q / 2\})$ is oriented from $i$ to $i^{\prime}$, then $\left\langle X / R_{0} \cup R_{1} \cup R_{2}\right\rangle$ is exactly the 3-edge presentation of $\Pi_{1}\left(M^{3}\right)$ associated to $\Lambda$, since $\tau_{0}, \tau_{1}, \tau_{2}$ are directly related with the 2 -residues of $\Lambda$ containing the colour 3.

\section{4. - Main results (dimension four).}

Let $(I, \gamma)$ be a crystallization of a closed connected orientable 4-manifold $M^{4}$, and let $V^{\prime}(T), V^{\prime \prime}(\Gamma)$ be the two bipartition classes of its vertex set. Further, let $\varepsilon=$ $=\left(\varepsilon_{0}, \varepsilon_{1}, \varepsilon_{2}, \varepsilon_{3}, \varepsilon_{4}\right)$ be an arbitrarily fixed permutation of the colour set. As in the previous section, for sake of notational simplicity, we shall always write $c$ instead of $\varepsilon_{c}$, for every $c \in \Delta_{4}$.

Let $(\tilde{\Gamma}, \tilde{\gamma})$ be the 5-coloured graph representing $M^{4}$ such that the pseudocomplex $K(\tilde{\Gamma})$ is obtained from $K(T)$ by baricentrically subdividing the tetrahedron opposite to the (unique) 4-labelled vertex $v_{4}$, and then by starring from $v_{4}$ itself. It is easy to check that $(\tilde{T}, \tilde{\gamma})$ can be directly obtained from $(I, \gamma)$ by means of the following rules:

- for every $v \in V(\Gamma)$, construct a copy of the 3-coloured graph $(G, g)$ of figure 5 , and label its vertices by the elements of the set $N_{24} \times\{v\}$, so that the vertex $(i, v)$ is the copy of the vertex $i$ of $(G, g)$;

- for every $i \in\{1, \ldots, 6\}$ (resp. $i \in\{7, \ldots, 12\}$ ) (resp. $i \in\{13, \ldots, 18\}$ ) (resp. $i \in\{19, \ldots, 24\})$, connect $(i, v)$ with $(i, w)$ by a 3-coloured edge iff $v$ and $w$ are connected in $(T, \gamma)$ by a 0-coloured (resp. 1-coloured) (resp. 2-coloured) (resp. 3-coloured) edge;

- for every $i \in N_{24}$, connect $(i, v)$ with $(i, w)$ by a 4-coloured edge iff $v$ and $w$ are connected in $(I, \gamma)$ by a 4 -coloured edge.

Let now $(\bar{\Gamma}, \bar{\gamma})$ denote the standard 5-coloured graph of figure 6 , which evidently represents $S^{4}$. An easy direct computation proves the following result.

Proposition 7. - Let $f:(\bar{\Gamma}, \bar{\gamma}) \rightarrow(\bar{\Gamma}, \bar{\gamma})$ be the map defined by

$$
f(i, v)= \begin{cases}A & \text { if } i \text { even and } v \in V^{\prime}(\Gamma), \\ B & \text { if } i \text { odd and } v \in V^{\prime}(\Gamma), \\ A^{\prime} & \text { if } i \text { even and } v \in V^{\prime \prime}(\Gamma), \\ B^{\prime} & \text { if } i \text { odd and } v \in V^{\prime \prime}(\Gamma) .\end{cases}
$$




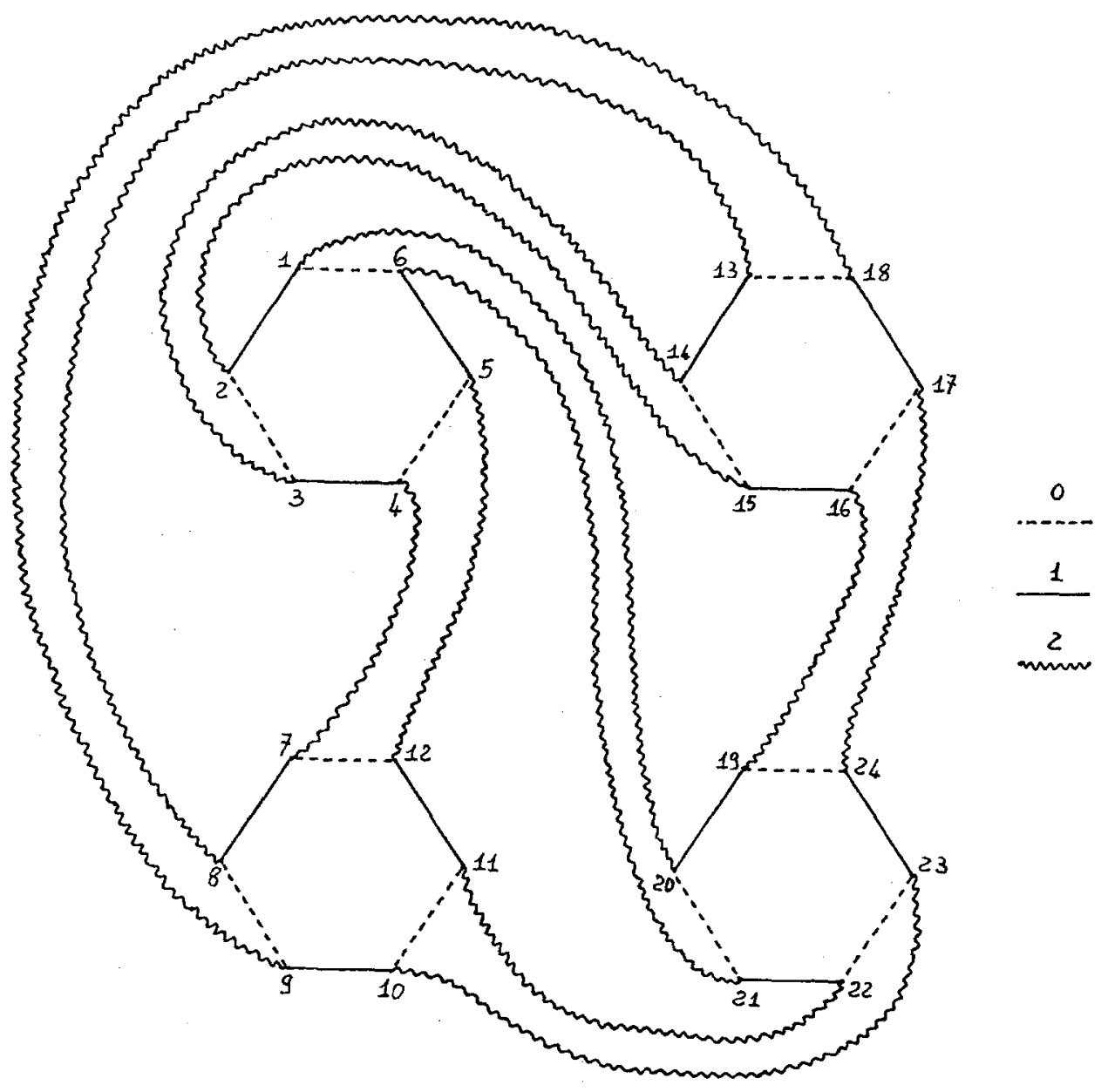

Fig. 5 .

Then, $f$ is a 1-covering, branched over the 2-residues of colours $\{0,1\},\{1,2\}\{0,2\}$, $\{2,3\},\{3,4\}$.

The 1-covering $f$ naturally induces a topological covering $|f|: M^{4} \rightarrow S^{4}$, branched over the subcomplex $K$ of $K(\bar{\Gamma})$ represented by the branching 2-residues of $(\bar{\Gamma}, \bar{\gamma})$. It is easy to check, by analizing the pseudosimplicial structure of $K(\bar{\Gamma})$, that the branching set $K$ is the bidimensional pseudocomplex depicted in figure 7 (where equally labelled vertices equally signed edges have to be identified).

Note that, in analogy with the branching set $G$ described in the previous section, $K$ contains exactly two 2 -spheres «connected» by one 2 -simplex, but it further contains an additional cone from a point of one sphere to a maximum circle of the other one. 


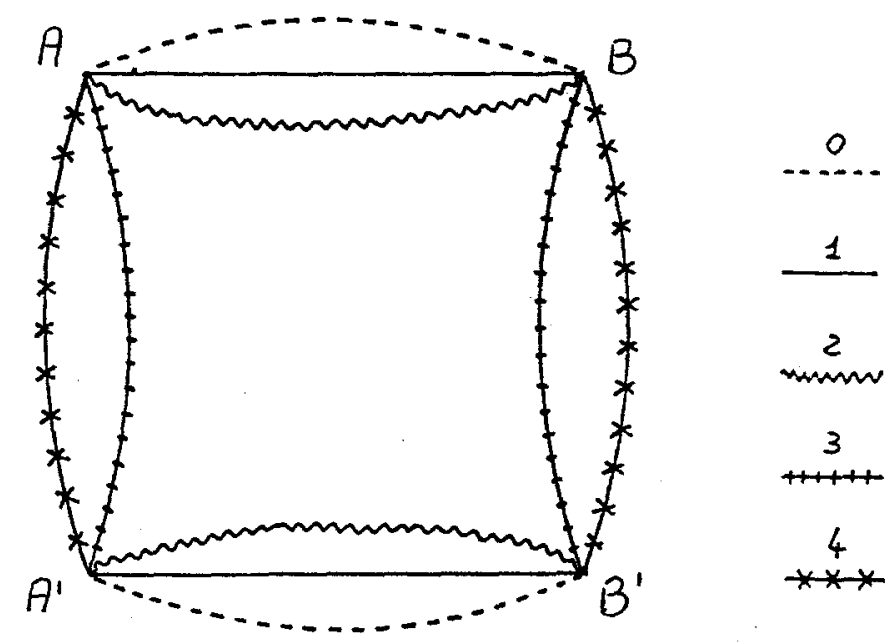

Fig. 6.

COROLLARY 8. - Every closed connected orientable 4-manifold $M^{4}$ is a covering of $S^{4}$ branched over the universal set $K$.

Since branched covering spaces are uniquely determined by the monodromy of the covering map [F], we are now going to determine the structure of the group of $K$, i.e. $\Pi_{1}\left(S^{4}-K\right)$. For, we consider the (unique) bisection $\bar{\Gamma}^{\prime}$ of type $(2,0)$ of $\bar{T}$ (see [G]) and then the (unique) bisection of type $(2,3)$ of $\bar{\Gamma}^{\prime}$; the resulting graph $\bar{\Gamma}^{\prime \prime}$ (which is drawn in figure 8) represents a pseudocomplex $K\left(\bar{\Gamma}^{\prime \prime}\right)$ triangulating $S^{4}$, such that $\left(K\left(\bar{\Gamma}^{\prime \prime}\right), K\right)$ is a branched 5 -coloured pair (with respect to the colour 2 ). Thus, it is possible to yield $\Pi_{1}\left(S^{4}-K\right)$ from the 2-edge presentation of $\Pi_{1}\left(S^{4}\right)$ associated to $\bar{\Gamma}^{\prime \prime}$.

Proposition 9. $-\Pi_{1}\left(S^{4}-K\right)=\left\langle a, b, c / a c a^{-1} c^{-1}=1\right\rangle$.

ProOF. - As pointed out in section $2, \Pi_{1}\left(S^{4}-K\right)$ is obtained from the 2-edge presentation of $\Pi_{1}\left(S^{4}\right)$ associated to $\bar{\Gamma}^{\prime \prime}$ by deleting all the relators corresponding to branching 2-residues (i.e. to 2-simplices of $K\left(\bar{\Gamma}^{\prime \prime}\right)$ belonging to the subcomplex $K$ ). Hence,

$$
\begin{aligned}
& \Pi_{1}\left(S^{4}-K\right)=\left\langle x_{1}, x_{2}, x_{3}, x_{4}, x_{5}, x_{6}, x_{7}, x_{8} / x_{5}=x_{6}, x_{7}=x_{8}, x_{5}=x_{8}\right. \\
& \left.\quad x_{6}=x_{7}, x_{1} x_{6} x_{4}^{-1} x_{7}^{-1}=1, x_{1}=x_{4}, x_{2}=x_{3}\right\rangle=\left\langle x_{1}, x_{2}, x_{5} / x_{1} x_{5} x_{1}^{-1} x_{5}^{-1} \stackrel{i}{=} 1\right\rangle .
\end{aligned}
$$

COROLlaRY 10. - Every closed connected orientable 4-manifold $M^{4}$ is represented by a suitable transitive set $\{\sigma, \tau, \mu\}$ of permutations, such that $\sigma \mu \sigma^{-1} \mu^{-1}=1$. 

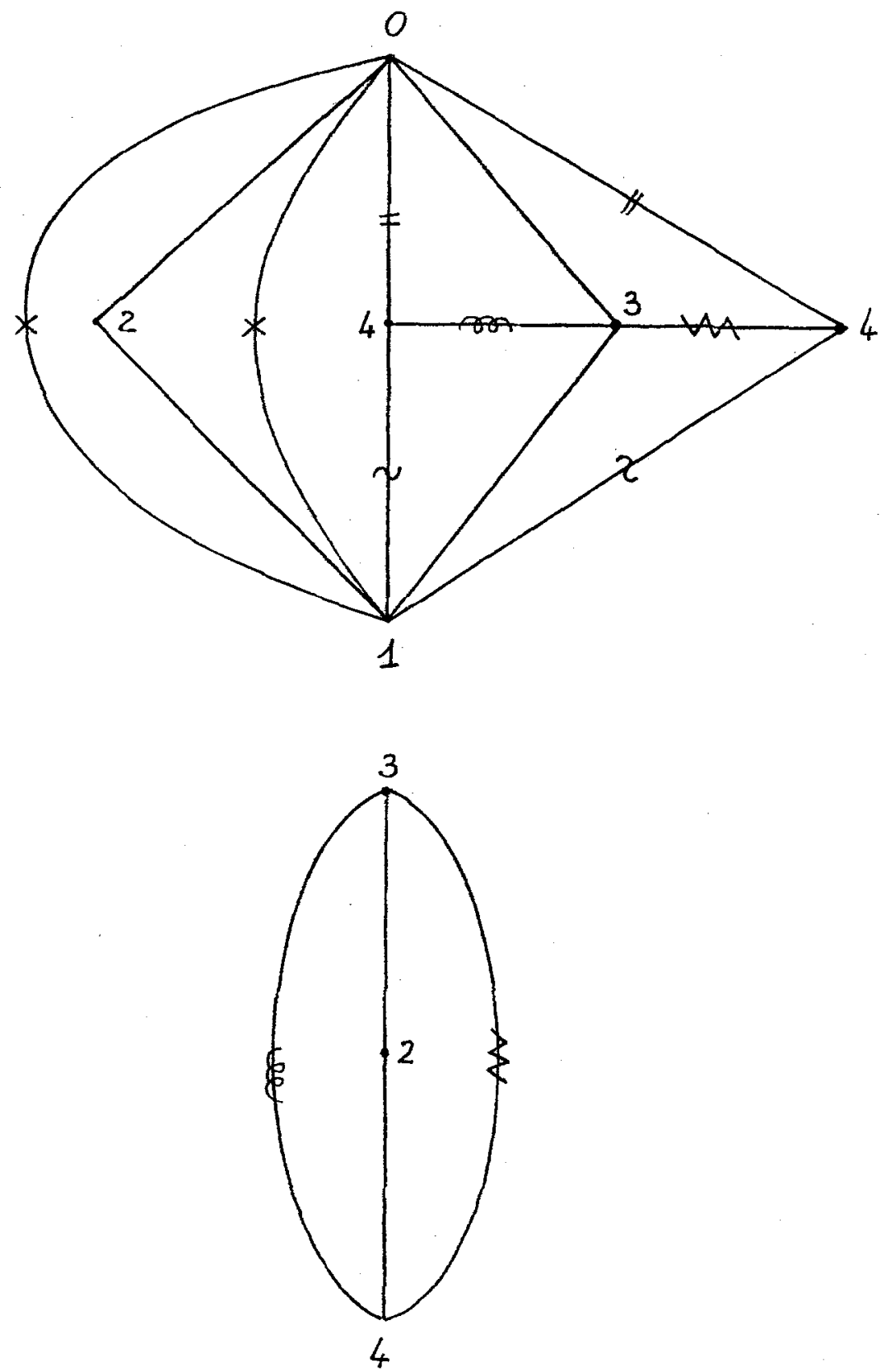

Fig. 7.

Proof. - If $\omega: \Pi_{1}\left(S^{4}-K\right) \rightarrow S_{d}$ is the monodromy associated to the covering map from $M^{4}$ to $S^{4}$ branched over $K$, it is sufficient to set $\sigma=\omega(\alpha), \tau=\omega(b), \mu=\omega(c)$, 


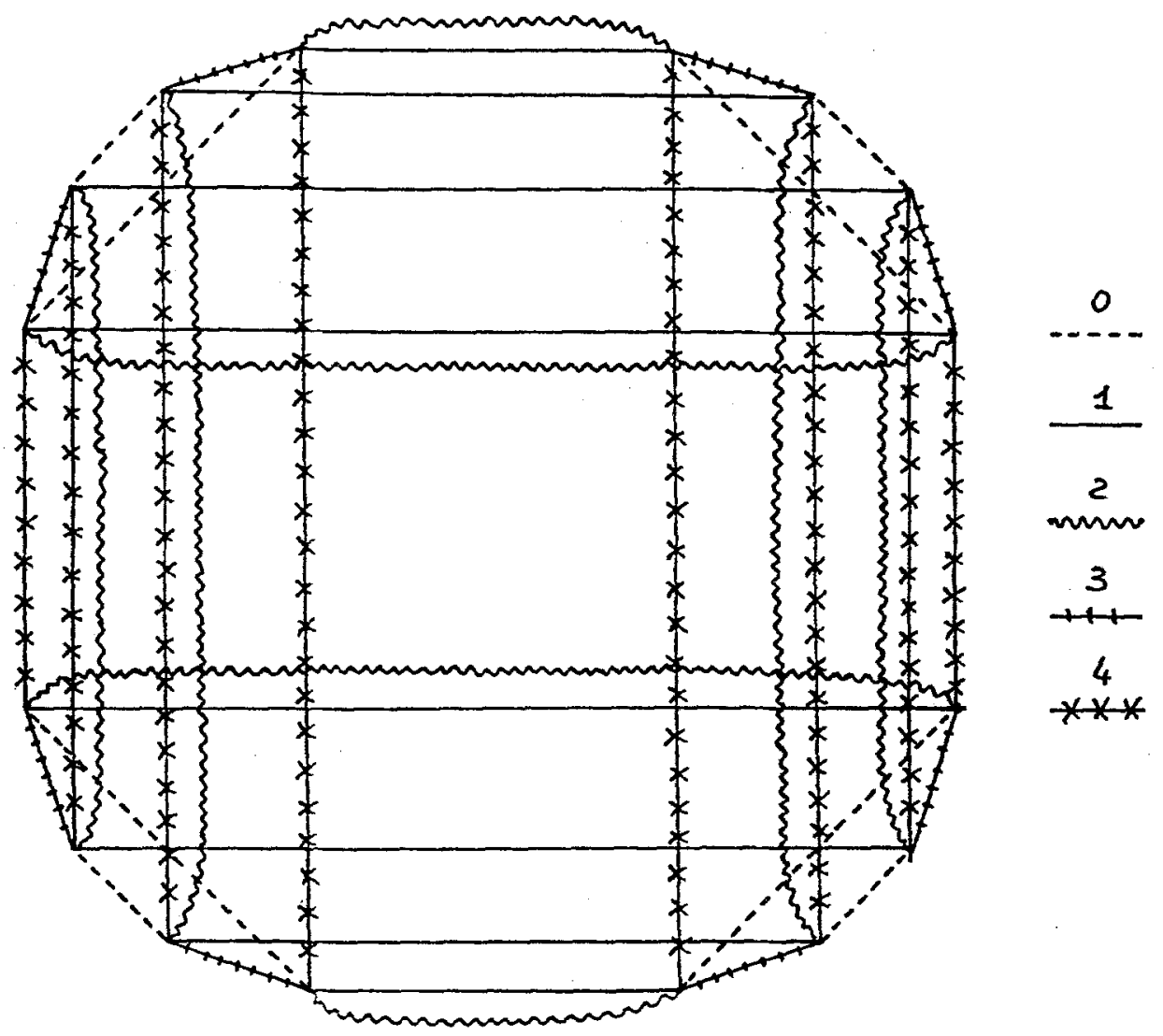

Fig. 8.

$a, b, c$ being the generators of the presentation of $\Pi_{1}\left(S^{4}-K\right)$ obtained in Prop. 9.

Given a transitive set $\{\sigma, \tau, \mu\}$ of permutations on $N_{d}$ such that $\sigma \mu \sigma^{-1} \mu^{-1}=1$, it is univocally determined the $d$-fold covering space $\Phi(\omega)$ of $S^{4}$ branched over $K$, $\omega: \Pi_{1}\left(S^{4}-K\right) \rightarrow S_{d}$ being the (unique) transitive representation extending the map $\psi:\{a, b, c\} \rightarrow S_{d}$ defined by $\psi(a)=\sigma, \psi(b)=\tau, \psi(c)=\mu$. However, it is easy to check that the 4-dimensional polyhedron $\Phi(\omega)$ is not necessarily a manifold.

We want now the analize the algebraic features of the set $\{\sigma, \tau, \mu\}$ of permutations associated to a given 4-manifold $M^{4}$, by computing the monodromy of the topological covering $|f|$ induced by the 1-covering $f$ of Prop. 7 . In order to do this, we consider the (global) bisection $\widetilde{\Gamma}^{\prime}$ of type $(2,0)$ of $\widetilde{\Gamma}$, and then the (global) bisection of type $(2,3)$ of $\widetilde{T}^{\prime}$, so that the 1-covering $f$ between $\widetilde{T}$ and $\bar{T}$ naturally induces a 1-covering $f^{\prime \prime}$ between the resulting graph $\widetilde{\Gamma}^{\prime \prime}$ (representing $M^{4}$ ) and the graph $\bar{\Gamma}^{\prime \prime}$ (representing $S^{4}$ ). It is now easy to check that the 1-covering $f^{\prime \prime}: \widetilde{\Gamma}^{\prime \prime} \rightarrow \bar{\Gamma}^{\prime \prime}$ can be obtained as an application of a standard method-due to [CG] and briefly described in section 2-to 
yield coloured graphs representing branched coverings of the sphere, starting from the associated monodromy. Since $\widetilde{\Gamma}^{\prime \prime}$ contains twelve copies of $\bar{\Gamma}_{\overline{2}}^{\prime \prime}$ for every pair $\{v, w\}$ of 4-adjacent vertices of $(\Gamma, \gamma), f^{\prime \prime}$ induces a topological $(12 \cdot p / 2)$-fold covering $(p=$ $=\# V(\Gamma))$ whose monodromy is described by the 2-coloured edges connecting the various sheets.

Thus, $\sigma, \tau, \mu$ may be thought of as permutations on the set $S=N_{12} \times N_{p / 2}$, by denoting $V^{\prime}(\Gamma)=\{1,2, \ldots, p / 2\}$ and $V^{\prime \prime}(\Gamma)=\left\{1^{\prime}, 2^{\prime}, \ldots, p^{\prime} / 2\right\}$, with $i$ 4-adjacent to $i^{\prime}$ for every $i \in N_{p / 2}$; moreover, the combinatorial properties of the graph $\tilde{\Gamma}^{\prime \prime}$ ensure that $\sigma$ and $\tau$ may always be assumed to be of standard type (only depending on $p=$ $=\# V(\Gamma)$ ), while $\mu$ directly depends on the combinatorial properties of the starting crystallization $(\Gamma, \gamma)$ of $M^{4}$ :

$\sigma=\prod_{i=1}^{p / 2}[((1, i)(2, i)(3, i))((4, i)(5, i)(6, i))((7, i)(8, i)(9, i))((10, i)(11, i)(12, i))]$, $\tau=\prod_{i=1}^{p / 2}[((1, i)(8, i)(10, i))((2, i)(4, i)(7, i))((3, i)(11, i)(6, i))((5, i)(12, i)(9, i))]$,

$$
\mu((x, i))=\left(x, \mu_{j(x)}(i)\right), \quad \text { where } j(x)= \begin{cases}0 & x \in\{1,2,3\}, \\ 1 & x \in\{4,5,6\}, \\ 2 & x \in\{7,8,9\}, \\ 3 & x \in\{10,11,12\}\end{cases}
$$

and $\mu_{j}(i)(j \in\{0,1,2,3\})$, for every vertex $i \in V^{\prime}(\Gamma)$ is the vertex $r \in V^{\prime}(\Gamma)$ which is 4-adjacent to the vertex $r^{\prime} \in V^{\prime \prime}(\Gamma) j$-adjacent to $i$.

Note that the above defined permutations $\sigma, \tau, \mu$ obviously verify condition $\sigma \mu \sigma^{-1} \mu^{-1}=1$; moreover, $\{\sigma, \tau, \mu\}$ is a transitive set, since $\widetilde{\Gamma}^{\prime \prime}$ is a connected graph.

Further, we point out that the only permutation $\mu$ on $S$ is sufficient to reconstruct $(\Gamma, \gamma)$. In fact, $(\Gamma, \gamma)$ is isomorphic to the 5-coloured graph $\Gamma(\mu)$ obtained by the following rules:

- the vertex set of $\Gamma(\mu)$ is $\{1,2, \ldots, p / 2\} \cup\left\{1^{\prime}, 2^{\prime}, \ldots, p^{\prime} / 2\right\}$;

- every vertex $i \in\{1,2, \ldots, p / 2\}$ is connected with the homonymous vertex $i^{\prime} \in\left\{1^{\prime}, 2^{\prime}, \ldots, p^{\prime} / 2\right\}$ by a 4 -coloured edge;

- every vertex $i \in\{1,2, \ldots, p / 2\}$ is connected with the vertex $\left(\mu_{j}(i)\right)^{\prime} \in\left\{1^{\prime}, 2^{\prime}, \ldots, p^{\prime} / 2\right\}$ by a $j$-coloured edge, for every $j \in\{0,1,2,3\}$.

Thus, we have described an algorithm for obtaining, from any 4-manifold $M^{4}$ - given by a crystallization $(T, \gamma)$-, a permutation $\mu$ which completely represents it; this leads to the following result. 
Proposition 11. - Every closed connected orientable 4-manifold $M^{4}$ is represented by a suitable permutation $\mu$ on $S=N_{12} \times N_{p / 2}$ such that

$$
\pi_{1} \circ \mu=1 \quad \text { and } \quad\left(\pi_{2} \circ \mu\right)((x, i))= \begin{cases}\mu_{0}(i) & x \in\{1,2,3\}, \\ \mu_{1}(i) & x \in\{4,5,6\}, \\ \mu_{2}(i) & x \in\{7,8,9\}, \\ \mu_{3}(i) & x \in\{10,11,12\},\end{cases}
$$

(or, equivalently, by a suitable set $\left\{\mu_{0}, \mu_{1}, \mu_{2}, \mu_{3}\right\}$ of permutations on $N_{p / 2}$ ).

REMARK. - The four permutations $\mu_{0}, \mu_{1}, \mu_{2}, \mu_{3}$ on $N_{p / 2}$ may be thought of as the rows of a «code» for 4-manifolds, extending the one defined in [LD] for dimension three.

Obviously, Prop. 11 can not be reversed: not every set $\left\{\mu_{0}, \mu_{1}, \mu_{2}, \mu_{3}\right\}$ of permutations on $N_{p / 2}(p \in N)$ is the result of the described algorithm applied to a $p$-vertices crystallization $(\Gamma, \gamma)$ of a 4-manifold $M^{4}$. Nay, we know no direct method to decide whether this happens or not. A partial answer is based on the combinatorial properties of the 5-coloured graph $\Gamma(\mu), \mu$ being obtained from $\left\{\mu_{0}, \mu_{1}, \mu_{2}, \mu_{3}\right\}$ by formula (\#).

Proposition 12. - Let $\mathscr{P}=\left\{\mu_{0}, \mu_{1}, \mu_{2}, \mu_{3}\right\}$ be a set of permutations on $N_{p / 2}$ $(p \in N)$. $\mathscr{P}$ represents a singular 4 -manifold $M^{4}$ if and only if $\Gamma(\mu)$ satisfies $(1) g_{\hat{c}}=1$ and (2)

$$
\sum_{j, k \in \Delta_{4}-\{c\}} g_{j k}-\sum_{j \in \Delta_{4}-\{c\}} g_{\hat{c} \hat{j}}=p \quad \text { for every } c \in \Delta_{4}
$$

Proof. - It is evident that $\mathscr{P}$ represents a singular 4-manifold $M^{4}$ if and only if $\Gamma(\mu)$ does, i.e. if its (unique) $\hat{c}$-residue $\Gamma_{\bar{c}}$ represents a closed 3 -manifold, for every $c \in \Delta_{4}$. Condition (2) now easily follows by computing the Euler characteristic of $K\left(\Gamma_{\hat{c}}\right)$ :

$X\left(K\left(\Gamma_{\bar{c}}\right)\right)=$

$$
=\sum_{j \in \Delta_{4}-\{c\}} g_{\hat{c} j}-\sum_{j, k \in A_{4}-\{c\}} g_{j k}+\# E\left(\Gamma_{\hat{c}}\right)-\# V\left(\Gamma_{\hat{c}}\right)=\sum_{j \in \Delta_{4}-\{c\}} g_{\hat{c} \hat{j}}-\sum_{j, k \in \mathcal{S}_{4}-\{c\}} g_{j k}+p .
$$

If $\mathscr{P}=\left\{\mu_{0}, \mu_{1}, \mu_{2}, \mu_{3}\right\}$ is a set of permutations representing a closed 4-manifold $M^{4}$, then the crystallization $\Gamma(\mu)$ of $M^{4}, \mu$ being obtained from $\left\{\mu_{0}, \mu_{1}, \mu_{2}, \mu_{3}\right\}$ by formula (\#), is said to be canonically associated to $\mathcal{P}$.

The following result shows how easily $\mathscr{P}=\left\{\mu_{0}, \mu_{1}, \mu_{2}, \mu_{3}\right\}$ may produce the fundamental group of the represented 4-manifold, via the 4-edge presentation of the canonically associated crystallization. 
Proposition 13. - Let $\mathscr{P}=\left\{\mu_{0}, \mu_{1}, \mu_{2}, \mu_{3}\right\}$ be a set of permutations on $N_{p / 2}(p \in N)$, representing a closed 4 -manifold $M^{4}$. Then, a (positive) presentation of $I_{1}\left(M^{4}\right)$ is

$$
\left\langle X / R_{0} \cup R_{1} \cup R_{2} \cup R_{3}\right\rangle,
$$

where the set $X=\left\{x_{1}, x_{2}, \ldots, x_{p / 2}\right\}$ of generators is in bijection with $N_{p / 2}$, and the set $R_{i}=\left\{r_{i}^{1}, r_{i}^{2}, \ldots r_{i}^{\sigma_{i}}\right\}$ of relators $\left(i \in \Delta_{3}\right)$ is in bijection with the set $\left\{c_{i}^{1}, c_{i}^{2}, \ldots c_{i}^{\sigma_{i}}\right\}$ of disjoint cycles of the permutation $\mu_{i}, r_{i}^{s}\left(s=1,2, \ldots, \sigma_{i}\right)$ being obtained by juxtapposing the generators corresponding to the ordered elements of $c_{i}^{s}$.

Proof. - Let $\Gamma=\Gamma(\mu)$ be the crystallization of $M^{4}$ canonically associated to the set $\mathscr{P}$ of permutations. By construction, the set of 4-coloured edges of $\Gamma$ is $\left\{x_{1}, x_{2}, \ldots, x_{p / 2}\right\}, i \in\{1,2, \ldots, p / 2\}$ and $i^{\prime} \in\left\{1^{\prime}, 2^{\prime}, \ldots, p^{\prime} / 2\right\}$ being the end-points of $x_{i}$, for every $i \in\{1,2, \ldots, p / 2\}$. If every edge $x_{i}(i \in\{1,2, \ldots, p / 2\})$ is oriented from $i$ to $i^{\prime}$, then $\left\langle X / R_{0} \cup R_{1} \cup R_{2} \cup R_{3}\right\rangle$ is exactly the 4-edge presentation of $\Pi_{1}\left(M^{4}\right)$ associated to $\Gamma$, since $\mu_{0}, \mu_{1}, \mu_{2}, \mu_{3}$ are directly related with 2-residues of $\Gamma$ containing colour 4.

\section{5. - A glance towards higher dimensions.}

Without going into depth about detailed proofs, we devote the present last section to show how to extend the procedures developed in the previous section to arbitrary dimension.

Let $(\Omega, \omega)$ be a crystallization of a closed connected orientable $n$-manifold $M^{n}$; w.l.o.g., we may fix the permutation $\varepsilon=(0,1, \ldots, n)$ of the colour set. Let $(\widetilde{\Omega}, \widetilde{\omega})$ be the $(n+1)$-coloured graph representing $M^{n}$ such that the pseudocomplex $K(\widetilde{\Omega})$ is obtained from $K(\Omega)$ by baricentrically subdividing the $(n-1)$-simplex opposite to the (unique) $n$-labelled vertex $v_{n}$, and then by starring from $v_{n}$ itself. The particular geometrical structure of $K(\bar{\Omega})$ induces particular combinatorial properties on the $(n+1)$ coloured graph $(\tilde{\Omega}, \tilde{\omega})$ : six;

a) for $i \in\{0,1, \ldots, n-3\}$, every $\{i, i+1\}$-residue of $(\widetilde{\Omega}, \widetilde{\omega})$ has valence

b) for $i, j \in\{0,1, \ldots, n-2\}, j \neq i \pm 1$, every $\{i, j\}$-residue of $(\widetilde{\Omega}, \widetilde{\omega})$ has valence four;

c) for $i \in\{0,1, \ldots, n-3\}$ (resp. $i \in\{0,1, \ldots, n-2\}$ ) every $\{i, n-1\}$-residue (resp. $\{i, n\}$-residue) of $(\widetilde{\Omega}, \widetilde{\omega})$ has valence four.

On the other hand, the valences of the $\{n-1, n-1\}$ - and $\{n-2, n\}$-residues of $(\bar{\Omega}, \widetilde{\omega})$ directly depend upon the incidence structure of $K(\Omega)$.

It is now easy to check the existence of a canonical 1-covering $f$ between $(\widetilde{\Omega}, \widetilde{\omega})$ and the standard $(n+1)$-coloured graph $(\bar{\Omega}, \bar{\omega})$ representing $S^{n}$ of figure 9 ; moreover. 


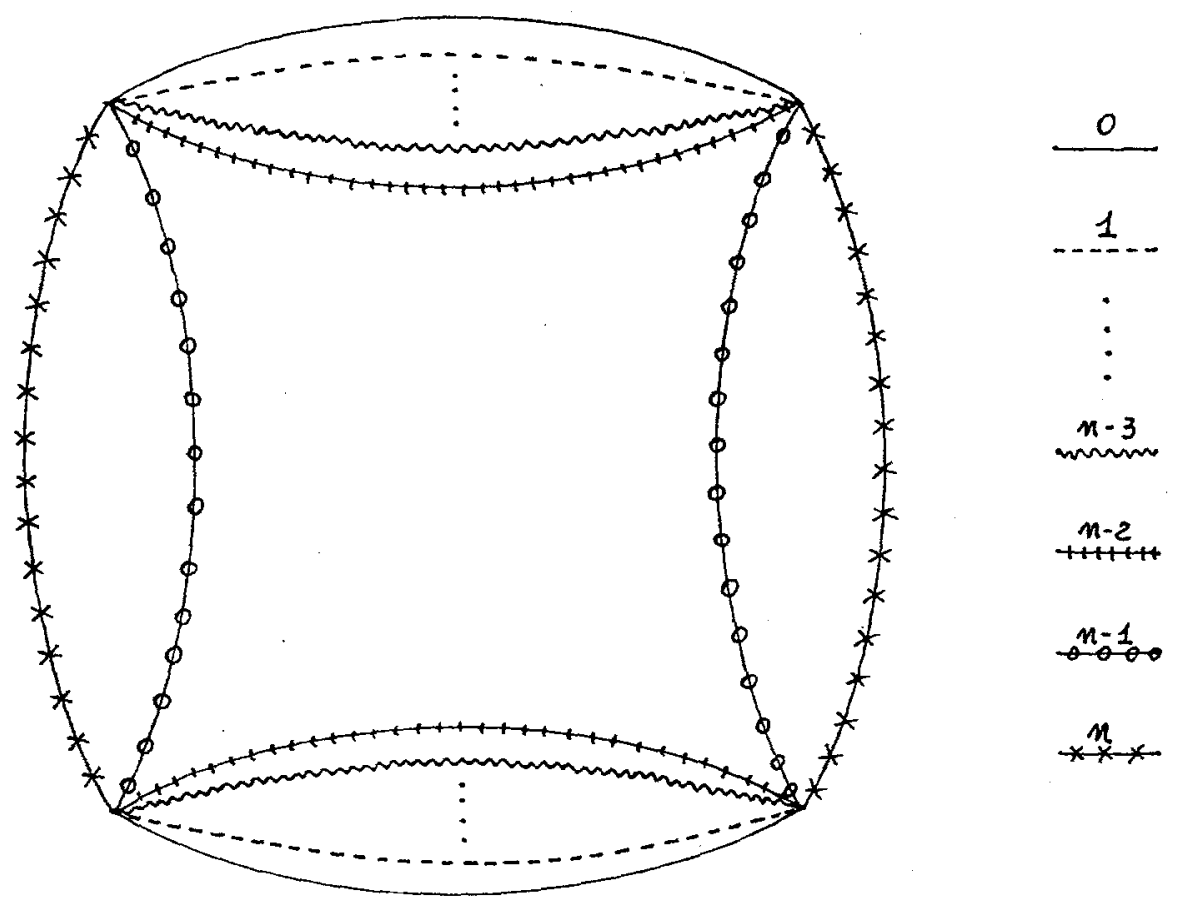

Fig. 9.

the branching 2-residues of $(\bar{\Omega}, \bar{\omega})$ are the $\{i, i+1\}$-residues, for $i \in\{0,1, \ldots n-3\}$ (with branching index three), the $\{i, j\}$-residues, for $i, j \in\{0,1, \ldots n-2\}, j \neq i \pm 1$ (with branching index two), the $\{n-2, n-1\}$ - and $\{n-1, n\}$-residues. Thus, the subcomplex $X=X^{n-2}$ of $K(\bar{\Omega})$ represented by the branching 2-residues of $(\bar{\Omega}, \bar{\omega})$ constitutes a universal branching set for $n$-manifolds.

Proposition 14. - Every closed connected orientable n-manifold $M^{n}$ is a covering of $S^{n}$ branched over $X^{n-2}$.

By performing on $(\bar{\Omega}, \bar{\omega}) n-2$ successive bisections (of type $(n-2, i)$, for $i=$ $=0,1, \ldots, n-4$ and finally for $i=n-1$ ), a new $\operatorname{graph} \bar{\Omega} *$ representing $S^{n}$ is obtained, such that $\left(K\left(\bar{\Omega}^{*}\right), X\right)$ is a branched $(n+1)$-coloured pair (with respect to the colour $n-2)$; thus, it is possible to yield $\Pi_{1}\left(S^{n}-X\right)$ from the $(n-2)$-edge presentation of $\Pi_{1}\left(S^{n}\right)$ associated to $\bar{\Omega}^{*}$ :

PROPOSITION 15. $\quad-\quad \Pi_{1}\left(S^{n}-X\right)=\left\langle x_{1}, x_{2}, \ldots, x_{2^{n-3}}, x_{2^{n-3}+1}=\bar{x} / x_{j} \bar{x}=\bar{x} x_{j}\right.$ $\left.\forall j \in\left\{1, \ldots, 2^{n-3}-1\right\}\right\rangle$.

HINT OF PROOF. - The presentation is obtained by considering, at first, all $(n-2)$ coloured edges of $\bar{\Omega}^{*}$ as generators, and then by reading the relations only from the non-branching 2-residues of $\Omega^{*}$, i.e. from the $\{i, n-2\}$-residues of $\bar{\Omega}^{*}$, for $i \in \Delta_{n}-$ 
$-\{n-1, n-2\}$, which are obtained by subdivision from the $\{i, n-1\}$-residues, for $i \in\{0, \ldots, n-3\}$, and the $\{j, n\}$-residues, for $j \in\{0, \ldots, n-2\}$, of $\bar{\Omega}$.

The following result is a direct consequence of the fact that branched covering spaces are uniquely determined by the monodromy of the covering map [F].

COROLlaRY 16. - Every closed connected orientable $n$-manifold $M^{n}$ is represented by a suitable transitive set $\left\{\sigma_{1}, \sigma_{2}, \ldots, \sigma_{2^{n-3}}, \sigma_{2^{n-3}+1}=\bar{\sigma}\right\}$ of permutations, such that $\sigma_{j} \bar{\sigma}=\bar{\sigma} \sigma_{j}, \forall j \in\left\{1, \ldots, 2^{n-3}-1\right\}$.

REMARK. - Corollary 16 holds for dimensions three and four, as well: in fact, for $n=3$ (resp. $n=4$ ) it reduces to Corollary 4 (resp. Corollary 10).

It is now possible to analize the algebraic features of the set $\left\{\sigma_{1}, \sigma_{2}, \ldots, \sigma_{2^{n-3}}, \sigma_{2^{n-3}+1}=\bar{\sigma}\right\}$ of permutations associated to a given $n$-manifold $M^{n}=$ $=K(\Omega)$, by means of the same techniques applied in sections three and four: by performing on $(\widetilde{\Omega}, \widetilde{\omega}) n-2$ successive bisections, a new $(n+1)$-coloured graph $\left(\widetilde{\Omega}^{*}, \widetilde{\omega}^{*}\right)$ is obtained, so that the induced 1-covering between $\left(\widetilde{\Omega}^{*}, \widetilde{\omega}^{*}\right)$ and $\left(\bar{\Omega}^{*}, \bar{\omega}^{*}\right)$ can be seen as an application of the construction described in [CG]. Thus, $\left\{\sigma_{1}, \sigma_{2}, \ldots, \sigma_{2^{n-3}}, \sigma_{2^{n-3}+1}=\bar{\sigma}\right\}$ may be thought of as the permutations defining the monodromy of a topological ( $p \cdot n ! / 4)$-fold covering $(p=\# V(\Omega))$; moreover, the combinatorial properties of the graph $\widetilde{\Omega}^{*}$ ensure that, for every $i \in\left\{1, \ldots, 2^{n-3}\right\}, \sigma_{i}$ may be assumed to be of standard type (only depending on $p=\# V(\Omega)$ and on the structure of any $\bar{n}$-residue of $(\widetilde{\Omega}, \widetilde{\omega})$, representing an $(n-1)$-simplex baricentrically subdivided), while $\bar{\sigma}$ directly depends on the combinatorial properties of the starting crystallization $(\Omega, \omega)$ of $M^{n}$.

\section{REFERENCES}

[BM] J. BRACHO - L. MONTEJANO, The combinatorics of colored triangulations of manifolds, Geom. Dedicata, 22 (1987), pp. 303-328.

[CG] M. R. CASALI - L. GRASSELLI, Representing branched coverings by edge-coloured graphs, Topology Appl., 33 (1989), pp. 197-207.

[CV] A. COSTA - P. DEL VAL-MeLus, Crystallizations and permutations representing 3-manifolds, Boll. U.M.I. (7), 1-B (1987), pp. 477-490.

[FGG] M. FERri - C. GaGliard - L. GRASSELLi, A graph-theoretical representation of $P L$ manifolds. A survey on crystallizations, Aequationes Mat., 31 (1986), pp. 121-141.

[F] R. H. Fox, Covering spaces with singularities, in Proc. Symposium in Honor of S. Lefschetz, Princeton Math. Series, 12 (1957), pp. 243-257.

[G] C. GAGLIARDI, On a class of 3-dimensional polyhedra, Ann. Univ. Ferrara, 33 (1987), pp. 51-88.

[G1] L. C. Glaser, Geometrical Combinatorial Topology, Van Nostrand Reinhold Math. Studies, New York (1970). 
[Gr] L. GRASSELLI, Edge-coloured graphs and associated groups, in Atti II Convegno Topologia, Taormina 1984, Rend. Circ. Mat. Palermo (2), 12 (1986), pp. 263-269.

[HW] P. J. HILTon - S. WYLIE, An Introduction to Algebraic Topology - Homology Theory, Cambridge Univ. Press, Cambridge (1960).

[LD] S. LINS - C. DURAND, A complete catalogue of rigid graph-encoded orientable 3-manifolds up to 28 vertices, Preprint UFPE - Recife 1989 (to appear).

[M] J. M. MonTEsInos, Representing 3-manifolds by a universal branching set, Math. Proc. Camb. Philos. Soc., 94 (1983), pp. 109-123.

[P] M. Pezzana, Sulla struttura topologica delle varietà compatte, Atti Sem. Mat. Fis. Univ. Modena, 23 (1974), pp. 269-277.

[RS] C. P. ROURKE - B. J. SANDERSON, Introduction to piecewise-linear topology, SpringerVerlag (1972).

[V] A. VINCE, Combinatorial maps, J. Comb. Theory Ser. B, 34 (1983), pp. 1-21.

[W] A. T. WhITE, Graphs, Groups and Surfaces, North-Holland (1973). 